\title{
CelGuider: A Low-cost Accessible Geographical Tagging System
}

\author{
Thais Melo \\ University of Washington \\ Seattle, WA, USA \\ thaismelo@gmail.com
}

\author{
Joyojeet Pal \\ ATLAS Institute \\ University of Colorado, Boulder, USA \\ joyojeet@gmail.com
}

\begin{abstract}
The growing field of technology and development has brought to the world's attention innovation in interfaces with regard to issues of social and economic inclusion, and yet there has been almost no work on accessibility in the developing world. This oversight arguably reflects the state of innovation in this space - despite research and development of several access tools to allow for greater physical navigation and social inclusion for persons with vision impairments, the prohibitive cost of such technology has restricted their use primarily to the developed world. With the range of communications and navigation options available through smart-phones, there is an opportunity to drastically reduce the cost of such access, in part off-the-shelf phones may offset much of what is offered by specialized devices. CelGuider explores navigation on foot and road vehicle transportation using location-tagging on a Google Android phone. This paper discusses the key design challenges for Low Vision and Blind users in the following areas: creating, updating, and storing tags at the phone, communicating audio information to the users, integrating GPS, power considerations, and the variability of tagging mechanics based on street architecture scenarios and proposes an interface for Low Vision and Blind users. Finally, we discuss the importance of much user testing for the contextual information necessary to understand individual and social practices around navigation, as well as cost factors for smart phones in the developing world.
\end{abstract}

Geographical tagging, assistive technology, human factors.

\section{INTRODUCTION}

Article 9 of the UN Convention on the Rights of Persons with Disabilities has important consequences for several member states from the developing world that have signed and ratified the convention. Within this is enshrined the right of access to transportation without barriers for people with disabilities. Accessible streets and transportation for people with disabilities remains a challenge through much of the world, though some legislative measures and a greater recognition on social exclusion in public spaces have led to transit accessibility measures (Carr, Lund et al. 1994; Wehman, Wilson et al. 1999; Lewyn 2000). Such measures include both infrastructural improvements for transit vehicles, and the development of new technology to aid mobility and navigation. Two issues in physical navigation for people with visual impairments include the shortrange sensing of obstacles, and the medium- to long-range navigation to transit destinations. Of these, the first primarily deals with architectural accessibility such as streets or platforms with edges, traffic lights with audio prompts, and can be further be partly managed through aids such as walking canes. In this paper, we discuss the latter case, of navigation over medium-range distances which is more dependent on route-planning. Technological navigation systems for persons with visual impairments have been used and researched since the 1990s (Golledge, Loomis et al. 1991) but such devices often cost thousands of dollars, and are often of the wearable computing kind which can be problematic in terms of bringing attention to users (Sasaki, Tateishi et al. 2000; Ran, Helal et al. 2001). As more innovations for people with disabilities turn to social models of disability, and thus take into account issues of the public gaze, there has been a growing interest in designing inconspicuous technologies (Williams, Greeff et al. 2008) or incorporating access-related features into existing personal technologies such as cell-phones to avoid the reliance on specialized technology (Cavender, Ladner et al. 2006). Global Positioning Systems based devices have created new possibilities for outdoor navigation, especially as their use has become more widespread and 
incorporated into commodity cellular phones. Although there has been work in this space for well over a decade (Makino, Ishii et al.; Dodson, Moon et al. 1999), there has been little exploration of the possibilities within the use of navigation systems in an environment of folksonomy-based tagging of locations (Mathes 2004). With a combination of GPS and basic geospatial data and a cellular device that can be used to tag locations and share such tags, a navigation system can be built on a basic bootstrap of data and provide users with general information about location, as well as route-specific information relevant to a user's routine. Such navigation systems may significantly expand access to transportation for users in several parts of the world, but this may require a number of pre-requisites such as the availability of reliable geo-spatial data, the existence of a relatively regular and physically accessible public transit system, affordability of smart phones, plus a range of cultural factors that may determine the usability of such devices. Indeed, many of these factors are currently unavailable in much of the developing world, but the expansion of access to geospatial data and the falling prices of technology suggest that it is important to start thinking in the direction of accessibility devices that run on off-theshelf components. We present CelGuider, a cellphone based navigation system that uses basic GPS data to provide location information and allows for tagging of locations. While currently prevalent infrastructure may not allow for the full use of CelGuider in many parts of the developing world, we argue that this will soon change as many countries, especially those that have ratified the optional protocol to the UN Convention, seek to increase accessibility on public transit. While work on the emerging field of "ICTD" has brought to fore a range of new technologies for social inclusion, there has been little work on accessibility (Kalra, Lauwers et al. 2007), an important failing of the community in addressing broad social inclusion. In the following sections, we present a detailed design description and the outcomes of trials held in Seattle to discuss some of the interface concerns and the potential of deployment in different scenarios in the developing world.

\section{CELGUIDER SOFTWARE}

The core idea of CelGuider is to allow users to create and delete tags correlating geographical locations to voice tags using a cell-phone. Once created, those tags can then be used to assist users in geographical navigation. When the tags are created using CelGuider, the geographical locations, in the form of longitude and latitude, are obtained via GPS and the voice tags are recorded by the user speaking into the phone.

\subsection{Target Users}

The target users for CelGuider have several of the following characteristics: they reside in developing countries; the locations they need to navigate have poor or no cellular network access; they have limited or intermittent access to electrical power; they do not have access to a computer and the Internet; they don't have the resources to acquire assistive technology tools available in the market; the locations they navigate or the languages they speak are not covered in other accessible navigating tools targeted at the developed world.

The particular user needs that CelGuider targets are independent geographical navigation and using public transit.

\subsection{Core Features}

User interaction with the software happens in two distinct modes - Browsing Mode and Navigation Mode. Navigation Mode alerts the user of approaching points of interest (POIs) and allows the tagging of POls. Browsing Mode allows the browsing and deletion of existing tags. By allowing users to add and remove tags using the cell-phone, CelGuider remains a standalone application, letting users maintain their tag lists without the need for any additional hardware or software.

\subsubsection{Navigation Mode}

CelGuider will start in Navigation Mode when launched. When entering Navigation Mode, the user will hear a notification ("Entering navigation mode.") using the Android text to speech API (TTS).

In Navigation Mode, CelGuider starts tracking the current location of the user in relation to all the tags currently present on the phone. This allows CelGuider to announce to the user, using the appropriate voice tag, when he is approaching a particular POI. There are two modes for notification, normal mode and motor vehicle transportation mode. In normal mode, the user is only notified when very close to the POI - 3 meters - to aid user in determining whether he is in the correct location. In motor vehicle transportation mode the user is notified $0.5 \mathrm{~km}$ away from the $\mathrm{POI}$, thus giving the user some advance notice the $\mathrm{POI}$ is approaching. CelGuider automatically transitions between the two modes based on the speed acquired by the GPS - it goes to motor vehicle transportation mode when the speed is higher than $10 \mathrm{~km} / \mathrm{h}$ and to normal mode when it is lower.

Navigation Mode also allows the user to add voice tags for his current location to facilitate future navigation. The user can press the " 0 " key on the 


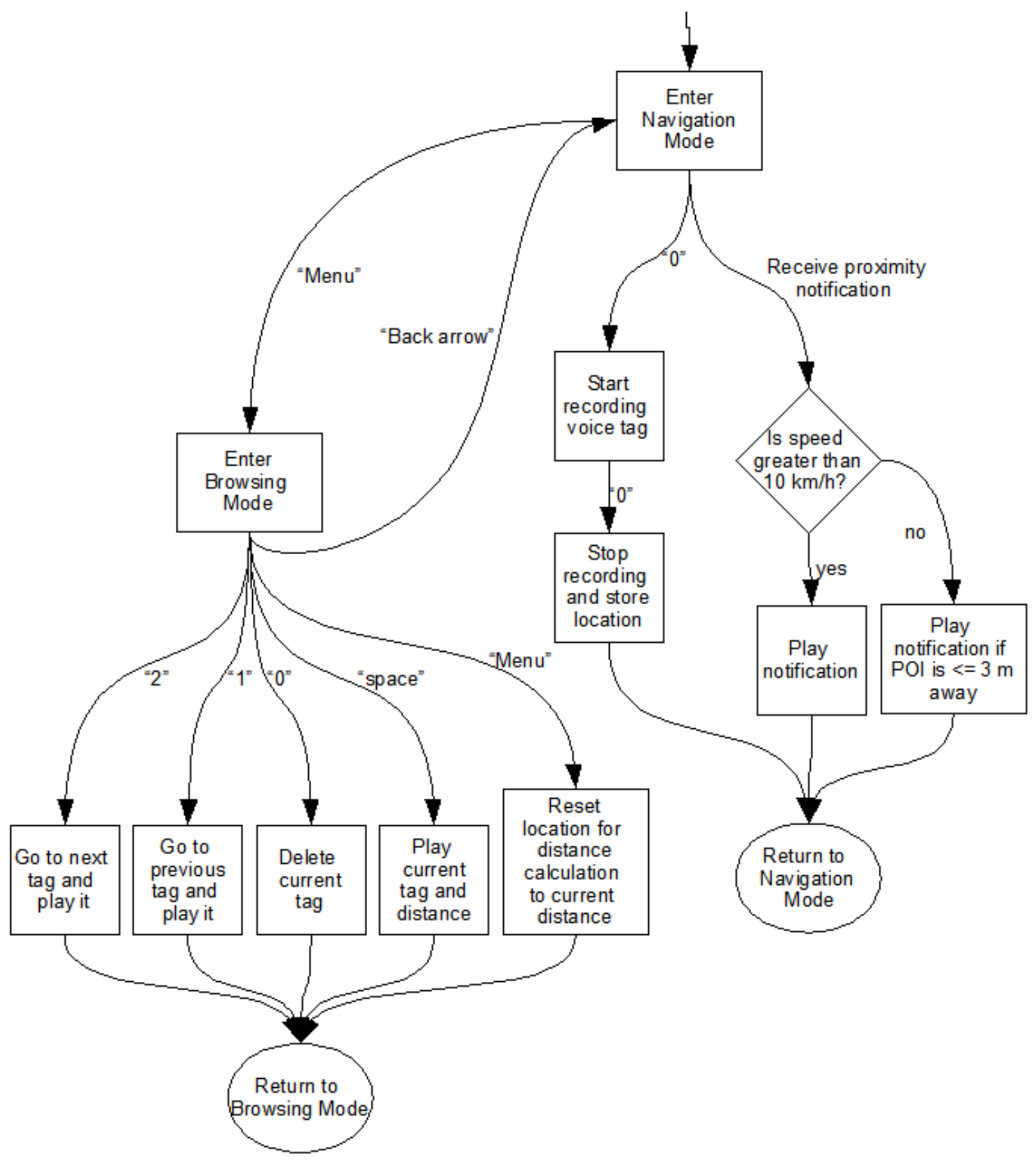

Figure 1: CelGuider user interface flow

keyboard to start recording a voice tag. When the user presses "0" again CelGuider stops recording, queries current location from the GPS, creates a database entry for the tag and stores the audio file with the user recorded voice tag.

The user can go to Browsing Mode by pressing the "Menu" key in the phone.

\subsubsection{Browsing Mode}

In Browsing Mode the user can browse through all tags available in order of distance. For each tag, the voice tag associated with it will be played, as well as the distance to the tagged geographical location.

When entering Browsing Mode, the user will hear a notification ("Entering browsing mode."). After this, 
the user can navigate between tags using the keyboard. The "1" key goes to the previous tag and the "2" key goes to the next tag. The tags are ordered by distance from the user's location when he entered Browsing Mode. The "Menu" key can be used to trigger a location reset and the reordering of tags by distance to the current location. The "space" key is used to repeat the audio of the current tag. In this mode, the user can also delete tags using the " 0 " key.

The user can go back to Navigation Mode by pressing the back arrow key in the device.

\subsection{Use case scenario}

The use case scenario we propose in this design uses CelGuider to navigate to a destination using on foot and public transportation. In this scenario, the user wants to go from location A to location B. He already knows the necessary information on how to go from location $A$ to location $B$ - in this example, the appropriate bus line and the correct direction of travel in the bus - and CelGuider has been bootstrapped with the appropriate bus stop locations. CelGuider can be used as follows:

(i) The user starts CelGuider at location A. The user presses the "Menu" key to go to Browsing Mode. In Browsing Mode the user can browse through tags to find the closest bus stop.

(ii) The user now presses the "back arrow" key to go back to Navigation Mode and proceeds to the bus stop. In Navigation Mode the user is notified when approaching the appropriate starting bus stop.

(iii) The user boards the bus. CelGuider is still in Navigation Mode and, due to the increased speed with which the user is traveling, enters motor vehicle transportation mode. In this mode, CelGuider notifies the user of the destination bus stop $0.5 \mathrm{~km}$ away from the stop, allowing the user time to alert the bus driver he wishes to leave before the target bus stop is passed.

(iv) When the user exits the bus, CelGuider goes back to normal mode as the user is either stopped or moving at walking speed. CelGuider issues the notification for the current bus stop, allowing the user to verify he exited the bus in the correct location.

(v) The user can now go to Browsing Mode and browse tags until he finds location $B$, and listen to the distance to location B.

(vi) The user goes back to Navigation Mode, and CelGuider notifies him when he arrives at location B.

\subsection{Testing}

Testing was done with 5 users with no visual disabilities. User testing led to several refinements of the prototype. Some examples are decreasing the notification distance in motor vehicle mode to 500 meters from $1 \mathrm{~km}$ and changing the order in which the tags and speed were spoken in Browsing Mode. One important aspect of our testing was that CelGuider has a very limited visual user interface. This avoids masking hidden accessibility problems in the software while testing with users with no visual disabilities ${ }^{1}$.

\subsubsection{Testing bootstrapped device with users} Testing was done for the use case scenario described in section 2.3 as follows:

- Researcher described CelGuider to the users and gave them instructions on how to use it;

- Researcher asked users to use CelGuider to assist them in accomplishing scenario described in section $2.3^{2}$;

- Researcher observed how users interacted with the software and was available to respond to any user questions.

Two main issues were found in testing this portion of the work. The first was that the distance at which we initially had set the motor vehicle notification to be played $(1 \mathrm{~km})$ was too far away from the final destination. This parameter was changed to $0.5 \mathrm{~km}$ after the first two testers gave us feedback that the interval was too long. The new distance received positive feedback from the three remaining testers. The second main issue was GPS precision for walking mode. For two testers CelGuider sounded the notification within a few meters of the correct location for start and end location, for one tester one point was sounded over 10 meters away from correct location and for two testers one of the end points did not receive a notification. All notifications sounded correctly in motor vehicle mode. The main qualitative feedback from users was that they found the motor vehicle mode particularly useful and that CelGuider was easy to use.

\subsubsection{Creating and managing tags}

For this portion of the test users were asked to create tags, browse through them and then delete them.

\footnotetext{
${ }^{1}$ In Browsing Mode the only UI visible to testers was a black screen with the application name. In Navigation Mode a list of files used by CelGuider was displayed. The list of files was only there for debugging purposes for the researcher, as the file names are random strings.

${ }^{2}$ Note that we use simulated bus stops and bus routes to facilitate testing - actual transportation was done in a car.
} 
Users had no trouble navigating through tags or creating and deleting them. The user feedback included switching the order in which the voice recordings and distances were spoken in Browsing Mode, playing the voice recording of the deleted tag when the user deletes a tag, adding a check to ask the user if he really wants to delete a tag, adding a mechanism to activate and deactivate particular tags and telling users how many tags are left after a tag is deleted.

\subsubsection{Creating a set of bootstrapping tags}

As stated previously, bootstrapping data is important to make CelGuider a more compelling application. Unlike previous tests, this test was run with only one user. The task was to, given the latitude, longitude and text for bus stop tags, create a set of bootstrapping tags for the major bus stops on the 545 bus line in Sound Transit.

To create the tags one needs to record the voice tags in 3gp format and to generate a Sqlite database that contains a table that maps the geographical locations to the file names for the voice recordings. The creation of the voice recordings is difficult to automate, but in most situations automation could be used to speed up the translation of location data from any particular standard format to the Sqlite database used by CelGuider. Sqlite databases follow standard formats, so a database created on a computer should be readable in an Android phone. If automation is not a concern, one can simply use CelGuider in the Android emulator. In the emulator, the current location of the device can be mocked, allowing the user to create tags for POIs in arbitrary locations.

For this test, the Android emulator was used to create the tags, which were then transferred to the device. The 545 route $^{3}$ has 19 major stops. The voice recording took approximately 15 seconds per tag on average. Entering the latitude and longitude for each bus stop took 13 seconds on average. Sanity checking the tags for location correctness and clarity of recording took approximately 10 minutes. The overall time to complete this task was 19 minutes. King County has approximately 300 bus lines, thus, assuming the 545 is a representative line, the expected time to create such bootstrapping data for King County should be in the order of 95 person hours.

\subsection{Prototype Device}

The cell-phone used for this prototype was the Android HTC G1. It was chosen because it fulfills all the hardware and API requirements of this project (voice recording, voice play back, TTS

\footnotetext{
${ }^{3}$ http://www.soundtransit.org/x11978.xml
}

conversion, GPS location acquisition, database access and storage) and because its SDK is free and open source.

The G1 also has fairly accessible hardware for blind and low-vision users, following several guidelines from AccessWireless.org (Burton 2009). Some examples of particular relevance to this project are: hardware keyboard with well separated and tactile keys; differently shaped buttons for key functions on the phone; raised dot in the "f" and "j" keys (this is a variation on the AccessWireless.org guide $^{4}$, which suggests raised dot on 5 for a numeric keyboard).

The G1 also has a large touch screen that supports gestures, which we don't explore in this project. However, there have been positive results of using touch screens plus gestures to increase accessibility to blind or low vision users ${ }^{4}$, and some of the key based navigation in CelGuider could potentially be replaced by more intuitive gestures (i.e. swipe finger right to go to next POI).

As of the writing of this design case study, the offthe-shelf cost for an Android G1 is approximately $\$ 350$.

\section{BOOTSTRAPPING CELGUIDER}

Although CelGuider provides an accessible interface for the user to create their own tags, CelGuider is more useful with a set of bootstrap tags. For example, during user testing, CelGuider was bootstrapped with tags for the test simulated bus stops. Currently the only mechanism to bootstrap CelGuider is to manually transfer the file containing the tags to the phone by copying it to the phone memory card. CelGuider will read any Sqlite database files present in the "/sdcard/CelGuider" directory and load the tags present on those files into the CelGuider tag database.

Due to time constraints, CelGuider currently does not implement an accessible mechanism to upload, download or share tags. As this significantly simplifies bootstrapping of CelGuider, two different types of tag sharing are explored below.

\subsection{Sharing Tags between Two Phones}

Sharing between two phones allows two users using CelGuider to share the locations they have previously tagged. This is a particularly compelling scenario because users could simply use the Ad$\mathrm{Hoc}$ Wi-Fi available in the G1, not requiring any extra equipment or infrastructure. Also, since the

\footnotetext{
${ }^{4}$ Product Guide for Wireless Customers who are Blind or Visually Impaired: http://www.accesswireless.org/product/visual.cfm
} 
data storage follows the Sqlite standard, if CelGuider is ported to other phones, this mode would still be available for sharing.

\subsection{Downloading a Set of Tags from a Well Know Provider}

This could be useful in scenarios where a mapping service, a government service or a non-profit produce tagging data for a particular set of geographical locations. For instance, the government could publish a file with tags for all polling locations for an election, public transportation stops, hospitals, etc.

There are different ways the user could download this data into the phone, but they all require some form of external infrastructure. Some options include posting the data to an accessible website that could be used on the phone, creating a tool that could download a feed of tags to the phone or a kiosk mechanism, where users could go to a certain physical location and copy files with tags to the memory card in their phone.

\section{CONCLUSIONS AND FUTURE WORK}

Our purpose in creating CelGuider as a proof of concept was to start a much-needed conversation on access devices for the visually impaired in developing regions. While we acknowledge that the current cost of high-end phones and state of infrastructure in most parts of the world severely limits the practical reach of such a device, there is nonetheless evidence that a significant population could be functionally served by CelGuider. For instance, urban areas in middle-income countries are served by functional public transportation, and furthermore, there is reasonable geospatial data available on the lay of the land in these cities. In such cases, CelGuider has the potential to immediately offer a viable alternative to very expensive custom-made devices. In terms of broader access, there is reason for optimism that smart-phones will get cheaper or that lower end phones will feature the hardware needed to enable CelGuider. Advances in GIS mapping should also broaden the availability of geospatial data, enabling CelGuider to have richer functionality, such as giving users detailed directions between tags.

CelGuider's focus on relying on off-the-shelf components for hardware, an open framework for the software, and a hybrid bootstrapped and community-populated content base makes it an important cost-efficient direction for access technologies in the future. As the UN Convention ensures that more states that sign on the dotted line on providing reasonable access for people with disabilities make serious strides towards their commitment to social inclusion, a re-examination of access technology is imperative in the IDID community.

\section{REFERENCES}

Carr, M., T. Lund, et al. (1994). "Cross-sector benefits of accessible public transport, Project report: Transport Research Laboratory."

Cavender, A., R. Ladner, et al. (2006). MobileASL:: intelligibility of sign language video as constrained by mobile phone technology, ACM.

Dodson, A., G. Moon, et al. (1999). "Guiding blind pedestrians with a personal navigation system." The Journal of Navigation 52(03): 330-341.

Golledge, R., J. Loomis, et al. (1991). "Designing a personal guidance system to aid navigation without sight: Progress on the GIS component." International Journal of Geographical Information Science 5(4): 373-395.

Kalra, N., T. Lauwers, et al. (2007). A Braille Writing Tutor to Combat Illiteracy in Developing Communities, Citeseer.

Lewyn, M. (2000). "Thou Shalt Not Put a Stumbling Block before the Blind: The Americans with Disabilities Act and Public Transit for the Disabled." Hastings Law Journal 52: 1037.

Makino, H., I. Ishii, et al. Development of navigation system for the blind using GPS and mobilephone combination.

Mathes, A. (2004). "Folksonomies-cooperative classification and communication through shared metadata." Computer Mediated Communication.

Ran, L., A. Helal, et al. (2001). Drishti: An integrated navigation system for visually impaired and disabled.

Sasaki, H., T. Tateishi, et al. (2000). Wearable computer for the blind-aiming at a pedestrian's intelligent transport system.

Wehman, P., K. Wilson, et al. (1999). "Removing transportation barriers for persons with spinal cord injuries: An ongoing challenge to community reintegration." Journal of Vocational Rehabilitation 13(1): 21-30.

Williams, Q., M. Greeff, et al. (2008). Designing future technologies for disabled people in a developing country.

Burton, D. (1999). "Product Evaluation - The Revolutionary New iPhone.", http://www.afb.org/afbpress/pub.asp?DoclD=aw10 0502 (December 2009) 